Revista de Metalurgia 50(2)

Abril-Junio 2014, e015

ISSN-L: 0034-8570

doi: http://dx.doi.org/10.3989/revmetalm.015

\title{
Predicción de la dureza de aleaciones Al-Cu-Zn en estado de colada y templado
}

\author{
José D. Villegas-Cárdenas ${ }^{\mathrm{a}, \bowtie}$, Maribel L. Saucedo-Muñoz ${ }^{\mathrm{b}}$, \\ Víctor M. López Hirata ${ }^{b}$, Héctor J. Dorantes Rosales ${ }^{b}$ \\ ${ }^{a}$ Universidad Politécnica del Valle de México, Departamento de Ingeniería, Av. Mexiquense \\ s/n Col. Villa Esmeralda Tultitlan, Cp. 54910, Edo. México \\ ${ }^{b}$ Instituto Politécnico Nacional (ESIQIE), Departamento de Ingeniería Metalúrgica, Av \\ Instituto Politécnico Nacional s/n, Col. Lindavista, México D.F., Cp. 07738, México \\ Autor para la correspondencia: Jdvc76@yahoo.com.mx
}

Enviado: 12 Julio 2013; Aceptado: 8 Febrero 2014

\begin{abstract}
RESUMEN: Este trabajo presenta una nueva metodología experimental y numérica para pronosticar la dureza de las aleaciones Al-Cu-Zn, tanto en estado de colada como solubilizadas y templadas, cuyas composiciones químicas se encuentren dentro de dos líneas limítrofes, representadas por dos ecuaciones. De cada una de las líneas limítrofes se seleccionaron ocho composiciones diferentes que se caracterizaron por metalografía óptica, microscopía electrónica de barrido, difracción de rayos $\mathrm{X}$ y dureza. Las fases en equilibrio a diferentes temperaturas se obtuvieron por medio del programa Thermo-Calc. De la caracterización microestructural y análisis de regresión se obtuvieron los diferentes cambios de fase y dos ecuaciones de dureza. Al combinar las ecuaciones de dureza con las ecuaciones limítrofes se puede deducir la dureza de cualquier aleación con composición química dentro de esta zona. Para comprobar la exactitud de este método, se pronosticó la dureza de aleaciones a partir de los datos de algunos artículos de otros investigadores; las durezas estimadas presentaron tan solo un error menor o igual al 7\%.
\end{abstract}

PALABRAS CLAVE: Aleaciones Al-Cu-Zn; Estado de colada; Predicción de dureza; Solubilizada y templada

Citation / Cómo citar este artículo: Villegas-Cárdenas, J.D., Saucedo-Muñoz, M.L., López Hirata, V.M., Dorantes Rosales, H.J. (2014) "Predicción de la dureza para aleaciones Al-Cu-Zn en estado de colada y templado". Rev. Metal. 50(2): e015. doi: http://dx.doi.org/10.3989/revmetalm.015.

\begin{abstract}
Prediction of hardness for Al-Cu-Zn alloys in as-cast and quenching conditions. This work presents a new experimental and numerical methodology in order to predict the hardness in the as-cast, and solution treated and quenched $\mathrm{Al}-\mathrm{Cu}-\mathrm{Zn}$ alloys. Chemical composition of alloys is located inside two straight lines represented by two equations. Eight different compositions were selected from each line. All the alloys were characterized for light microscope, scanning electron microscope, X-ray diffraction and Rockwell B hardness test. The equilibrium phases were obtained at different temperatures by Thermo-Calc. The microstructure characterization and regression analysis enabled to determine the phase transformations and two equations of hardness assessment. The combination of hardness equations and composition line equations permitted to estimate the hardness of any alloy composition inside this zone. This was verified by calculating hardness with the information reported in other works, with an error lower than $7 \%$ in the estimated hardness.
\end{abstract}

KEYWORDS: Al-Cu-Zn alloys; As-cast; Hardness assessment; Solution treated and quenched

Copyright: $(\mathbb{C} 2014$ CSIC. This is an open-access article distributed under the terms of the Creative Commons AttributionNon Commercial (by-nc) Spain 3.0 License. 


\section{INTRODUCCIÓN}

Las aleaciones $\mathrm{Al}-\mathrm{Cu}-\mathrm{Zn}$ han sido ampliamente usadas debido a su alta resistencia a la tensión, corrosión y baja densidad, lo cual es una ventaja entre otro tipo de aleaciones, en la utilización de la industria aérea (Bai-quing, 1999). Lo anterior resalta la importancia de analizar las transformaciones de fase en el sistema ternario Al-Cu-Zn, el cual a temperatura ambiente presenta ciertas discrepancias entre algunos autores como Villars et al., (1997) y Börstein (2005). El primero sugiere que las fases que se presentan a $200{ }^{\circ} \mathrm{C}$ son las mismas que las que se presentan a temperatura ambiente, mientras que el segundo presenta fases muy diferentes a temperatura ambiente.

De acuerdo con Börnstein, las composiciones que se estudiaron en este trabajo comprenden tres zonas compuestas por las fases $\eta, \alpha$ y $\tau$ en la primera, la segunda por las fases $\tau$ y $\alpha$, la última compuesta por las fases $\tau, \theta$ y $\alpha$.

Existen discrepancias en algunas fases, pero con respecto a la fase $\tau$, no hay duda de su presencia; de acuerdo a varios investigadores esta fase debe estar presente (Sandoval et al., 2000; Zhu et al., 2001; Aragón et al., 2007a).

Se han realizado muchos estudios de las fases de equilibrio del sistema ternario $\mathrm{Al}-\mathrm{Cu}-\mathrm{Zn}$ debido a su importancia técnica (Malvano et al., 1911; Mondolfo, 1976; Ghosh et al., 1992), sin embargo, existen discrepancias entre los resultados de los estudios referenciados. Por tal motivo en este trabajo se utilizó el programa Thermo-Calc, para realizar un análisis termodinámico, de las fases que se deben encontrar en estado de equilibrio, de acuerdo a su temperatura. Lo anterior sirve para poder ver los diferentes cambios que se producirán en cada una de las composiciones conforme al tratamiento térmico utilizado (Wu et al., 2007).

El conocimiento de las fases presentes de este sistema servirá para entender las propiedades mecánicas en las aleaciones. Este conocimiento es siempre importante especialmente para partes estructurales, las cuales no son adecuadas para ser sujetas a una prueba convencional de tensión o compresión (Chang, 1976; Cenoz et al., 2007).

También dentro del sistema ternario Al-Cu-Zn se encuentran las aleaciones base $\mathrm{Zn}$ las cuales han mostrado buenas propiedades para ser usadas como cojinetes. Este tipo de aleaciones se han utilizado durante muchos años a pequeña escala y han demostrado tener excelentes propiedades tribológicas (Savaskan et al., 1983; Savaskan et al., 2003).

Se sabe que el $\mathrm{Cu}$ aumenta la dureza de estas aleaciones (Gelfi et al., 2004; Savaskan et al., 2004) y en investigaciones previas a este trabajo, se ha encontrado que existe una relación casi lineal entre la dureza y el porcentaje atómico del $\mathrm{Cu}$ (Villegas et al., 2011). Conforme a esta última investigación se ha planteado en este trabajo desarrollar dos series (ocho muestras de diferente composición en cada serie), las cuales cubrirán una cierta área del diagrama ternario Al-Cu-Zn (Fig. 1), que tiene relevancia debido a que dentro de esta zona se encuentra la zona eutéctica, que ha sido objeto de interés debido a sus propiedades superplásticas (Cáceres et al., 1987; Cáceres et al., 1990; Ramos et al., 2012).

El objetivo de este trabajo es desarrollar una metodología experimental que pronostique la dureza de cualquier aleación $\mathrm{Al}-\mathrm{Cu}-\mathrm{Zn}$ que esté dentro de esta zona, en función de su tratamiento térmico.

\section{MATERIALES Y MÉTODOS}

Se prepararon ocho composiciones diferentes de aleaciones $\mathrm{Al}-\mathrm{Cu}-\mathrm{Zn}$ de cada serie, donde las muestras designadas como M1 a M8 pertenecen a la serie 1 y de M9 a M16 a la serie 2 (Tabla 1). Cada una de estas series se representan por una línea recta definida por las ecuaciones:

$$
\begin{aligned}
& X_{Z n}=-1,9438\left(X_{C u}\right)+0,50334 \\
& X_{Z n}=-2,9823\left(X_{C u}\right)+0,97337
\end{aligned}
$$

donde $\mathrm{X}_{\mathrm{Zn}} \mathrm{y}_{\mathrm{Cu}}$ son las fracciones atómicas de $\mathrm{Zn}$ y $\mathrm{Cu}$, respectivamente. La composición química de cada una de las aleaciones utilizadas se presenta en la Tabla 1.

Cada una de las muestras se preparó por colada convencional con elementos metálicos puros a $750{ }^{\circ} \mathrm{C}$ bajo una atmósfera de argón y enfriados lentamente en el horno. Posteriormente, se sometieron a un proceso de homogeneización para eliminar la estructura dendrítica, durante 180 horas a $350{ }^{\circ} \mathrm{C}$ y se enfriaron lentamente en el horno. Por último, las muestras se volvieron a calentar a $350^{\circ} \mathrm{C}$ durante 24 horas y se enfriaron rápidamente a $2{ }^{\circ} \mathrm{C}$ en agua con hielo, con la finalidad de retener las estructuras cristalinas.

Las muestras así obtenidas se cortaron en tamaños de $2 \times 2 \times 1 \mathrm{~cm}$ y se prepararon utilizando técnicas metalográficas convencionales. Posteriormente fueron atacadas químicamente con una solución compuesta por $0,5 \mathrm{~cm}^{3}$ de ácido fluorhídrico y $99,5 \mathrm{~cm}^{3}$ de alcohol destilado. Posteriormente, las probetas pulidas se examinaron utilizando microscopía óptica y Microscopía Electrónica de Barrido (MEB). La composición en fases cristalinas se determinó mediante Difracción de Rayos X (XRD) con una radiación $\mathrm{K} \alpha$ de cobre. La dureza Rockwell "B" se determinó siguiendo procedimientos estándar. 
TABla 1. Composición química de las muestras estudiadas

\begin{tabular}{|c|c|c|c|c|c|c|c|c|c|c|c|c|c|}
\hline \multirow[b]{2}{*}{ Muestra } & \multicolumn{2}{|c|}{$\mathrm{Cu}$} & \multicolumn{2}{|c|}{ Zn } & \multicolumn{2}{|c|}{ Al } & \multirow[b]{2}{*}{ Muestra } & \multicolumn{2}{|c|}{$\mathrm{Cu}$} & \multicolumn{2}{|c|}{ Zn } & \multicolumn{2}{|c|}{ Al } \\
\hline & \%peso & \%at. & \%peso & $\%$ at & \%peso & \%at. & & \%peso & \%at. & \%peso & \%at. & \%peso & \%at. \\
\hline M1 & 5,00 & 3,54 & 63,18 & 43,44 & 31,82 & 53,02 & M9 & 5,00 & 4,77 & 89,60 & 83,10 & 5,40 & 12,13 \\
\hline M2 & 10,00 & 6,87 & 55,31 & 36,96 & 34,70 & 56,17 & M10 & 10,00 & 9,02 & 80,32 & 70,42 & 9,68 & 20,56 \\
\hline M3 & 15,00 & 10,03 & 47,43 & 30,82 & 37,57 & 59,15 & M11 & 15,00 & 12,83 & 71,04 & 59,05 & 13,96 & 28,12 \\
\hline M4 & 20,00 & 13,01 & 39,56 & 25,02 & 40,44 & 61,97 & M12 & 20,00 & 16,26 & 61,76 & 48,81 & 18,24 & 34,93 \\
\hline M5 & 25,00 & 15,84 & 31,69 & 19,52 & 43,31 & 64,64 & M13 & 25,00 & 19,37 & 52,48 & 39,52 & 22,52 & 41,01 \\
\hline M6 & 30,00 & 18,53 & 23,82 & 14,30 & 46,19 & 67,17 & M14 & 30,00 & 22,20 & 43,19 & 31,07 & 26,81 & 46,72 \\
\hline M7 & 35,00 & 21,08 & 15,94 & 9,33 & 49,06 & 69,59 & M15 & 35,00 & 24,79 & 33,91 & 23,35 & 31,09 & 51,86 \\
\hline M8 & 40,00 & 23,51 & 8,07 & 4,61 & 51,93 & 71,88 & M16 & 40,00 & 27,17 & 24,63 & 16,26 & 35,37 & 56,57 \\
\hline
\end{tabular}

\section{RESULTADOS Y DISCUSIÓN}

\subsection{Diagramas de equilibrio obtenidos mediante Thermo-Calc}

La Figura 1 muestra las diferentes fases que aparecen en el enfriamiento (de $400^{\circ}$ hasta $20^{\circ} \mathrm{C}$ ) de cada una de las muestras estudiadas. En la Tabla 2, se recoge, en función de la temperatura, una descripción de las fases más significativas. A temperatura ambiente, aparecen, para casi todas las muestras estudiadas, las fases $\tau, \alpha$ y $\eta$. Los resultados obtenidos, aplicando el programa Thermo Calc, indican que desde $200{ }^{\circ} \mathrm{C}$ a $20^{\circ} \mathrm{C}$ no existe ningún cambio de fases. A partir de los $200{ }^{\circ} \mathrm{C}$ las fases tienden a cambiar, apareciendo mayores transformaciones de fase en aquellas muestras que contienen una mayor cantidad de $\mathrm{Cu}$.

La muestra M11 presenta las mismas fases ( $\tau, \alpha$ y $\eta$ ) en el intervalo de temperaturas comprendido entre $400{ }^{\circ} \mathrm{C}$ y $20{ }^{\circ} \mathrm{C}$, lo que explica que el incremento de dureza entre colada homogénea y templada sea despreciable. El resto de las muestras presentan cambios continuos en sus fases en el intervalo de temperaturas indicado.

En el intervalo de temperaturas comprendido entre $300{ }^{\circ} \mathrm{C}$ y $20^{\circ} \mathrm{C}$, se observa que las muestras de la serie 1 (M1 a M8) presentan mayores cambios en sus fases que las de la serie 2 (M10 y M11), que permanecen con las mismas fases. Esto implica que las muestras de la serie 1 tendrán un mayor incremento de su dureza al momento de ser templadas que en el caso de las muestras de la serie 2.

Por otra parte los cálculos realizados con el programa Thermo-Calc permitieron obtener los datos necesarios para realizar un estudio comparativo entre los diagramas de Villars et al., (1997) y Börstein (2005). Se puede observar que el diagrama de Börstein se ajusta perfectamente a la simulación.
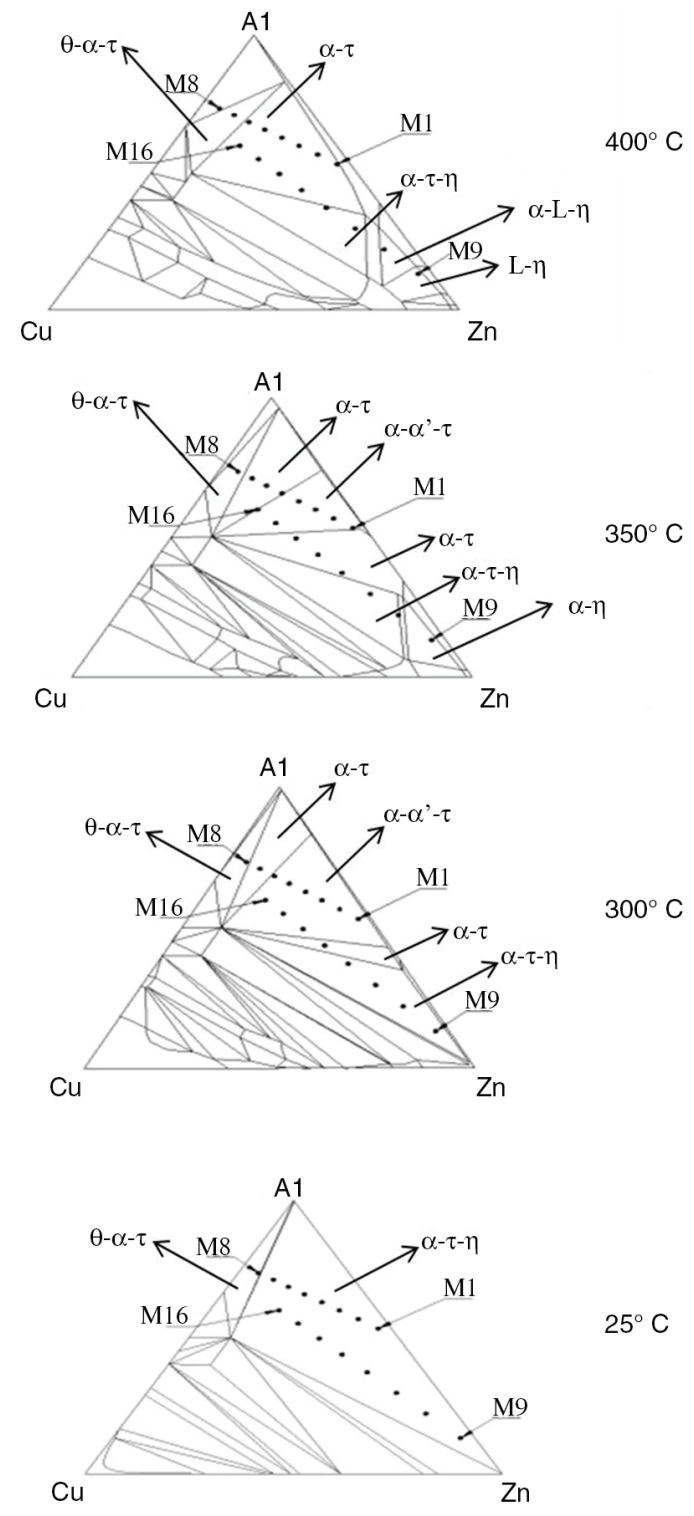

Figura 1. Resultados de la simulación en Thermo-Calc. 
TABLA 2. Fases presentes a diferentes temperaturas (obtenidas con el programa Thermo-Calc)

\begin{tabular}{lcclcc}
\hline Fases & Temperatura $\left({ }^{\circ} \mathbf{C}\right)$ & Muestra & Fases & Temperatura $\left({ }^{\circ} \mathbf{C}\right)$ & Muestra \\
\hline$\tau, \alpha$, & 400 & M1-M6 & L, $\eta$, & 400 & M9 \\
$\theta, \alpha, \tau$, & 400 & M7-M8 & L, $\alpha, \eta$, & 400 & M10 \\
& & $\tau, \alpha, \eta$, & 400 & M11-M12 \\
& & $\theta, \alpha, \tau$ & 400 & M13-M16 \\
$\tau, \alpha, \alpha^{\prime}$, & 350 & M1 a M4 & $\alpha, \eta$, & 350 & M9 \\
$\tau, \alpha$, & 350 & M5-M7 & $\tau, \alpha, \eta$, & 350 & M10-M11 \\
$\tau, \alpha, \theta$, & 350 & M8 & $\tau, \alpha$, & 350 & M15 \\
& & & $\tau, \alpha, \alpha$, & 350 & M16 \\
$\tau, \alpha, \alpha$, & 300 & & $\tau, \alpha$, & 350 & M9-M12 \\
$\tau, \alpha$, & 300 & M1-M5 & $\tau, \alpha, \eta$, & 300 & M13 \\
$\tau, \alpha, \theta$, & 300 & M6-M7 & $\tau, \alpha$, & 300 & M14-M16 \\
$\tau, \alpha, \eta$, & 25 & M8 & $\tau, \alpha, \alpha$, & 300 & M9-M16 \\
$\tau, \alpha, \theta$, & 25 & M1-M7 & $\tau, \alpha, \eta$, & 25 & \\
\hline
\end{tabular}

\subsection{Caracterización estructural y microestructural de las aleaciones}

Los difractogramas de las muestras en estado de colada, para cada serie, se muestran en la Figura 2. En los difractogramas de las muestras de la serie 1 (Fig. 2a) se puede observar que en casi todas las muestras se presentan las fases $\eta, \alpha, \varepsilon, \tau, \theta, \beta$. Una fase adicional $\mathrm{AlCu}$ se presenta únicamente en las muestras M6 y M7. Solo la muestra M8 presenta únicamente tres fases: $\eta, \alpha$ y $\theta$.

En el caso de las muestras de la serie 2 (Fig. 2b) se observa que las fases $\eta, \alpha, \varepsilon$ y $\tau$ están presentes en todas las muestras. Las fases $\theta$ y $\beta$ sólo aparecen en algunas muestras (M9 y de la M14 a M16). Cabe resaltar que la fase $\theta$ presenta una relación proporcional en cuanto al aumento de la intensidad de sus picos con respecto al aumento del contenido $\mathrm{Cu}$ y Al.

Las micrografías MEB de las muestras M2, M7, M10 y M15 se recogen en la Figura 3. Se observa un aumento de la fracción volumétrica de la fase $\theta$ con respecto al aumento del contenido de $\mathrm{Cu}$ y $\mathrm{Al}$, como se había puesto de manifiesto anteriormente. También se puede observar que las muestras con bajos contenidos de $\mathrm{Cu}$ y $\mathrm{Al}$ poseen una microestructura muy heterogénea, mientras que a mayor cantidad de $\mathrm{Cu}$ y $\mathrm{Al}$ el aspecto es más uniforme.

La Figura 4, recoge los difractogramas de las muestras después del proceso de homogeneizado. Se puede observar que en todas las muestras, tanto de la serie 1 como de la serie 2 , se encuentran presentes las fases $\eta, \alpha$, y $\tau$, en concordancia con los diagramas de equilibrio obtenidos por el programa
Thermo-Calc. Debido a que estas muestras no han alcanzado su estado de equilibrio, se puede observar que en todas ellas existen otras fases $(\varepsilon, \theta$ y $\beta)$.

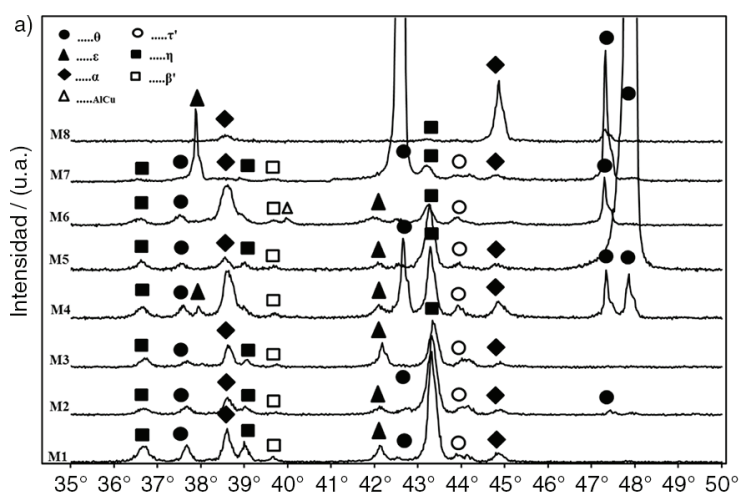

$2 \theta$

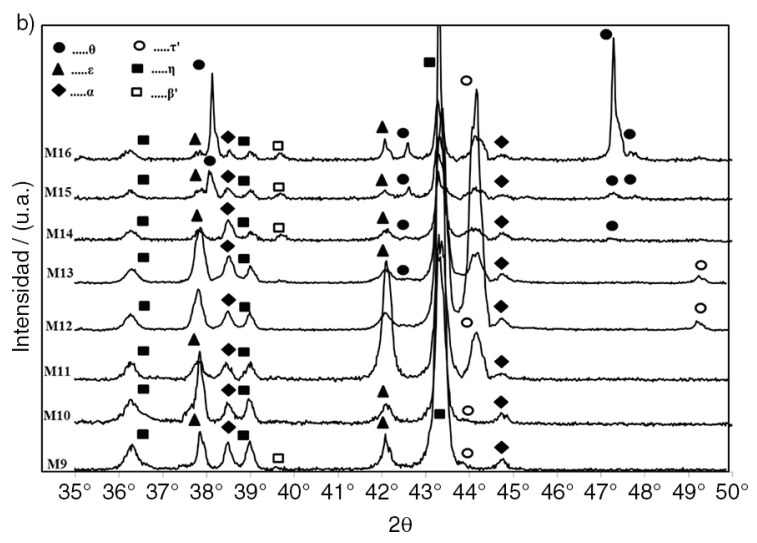

Figura 2. Difractogramas de las muestras en estado de colada. a) Serie 1 y b) Serie 2 . 

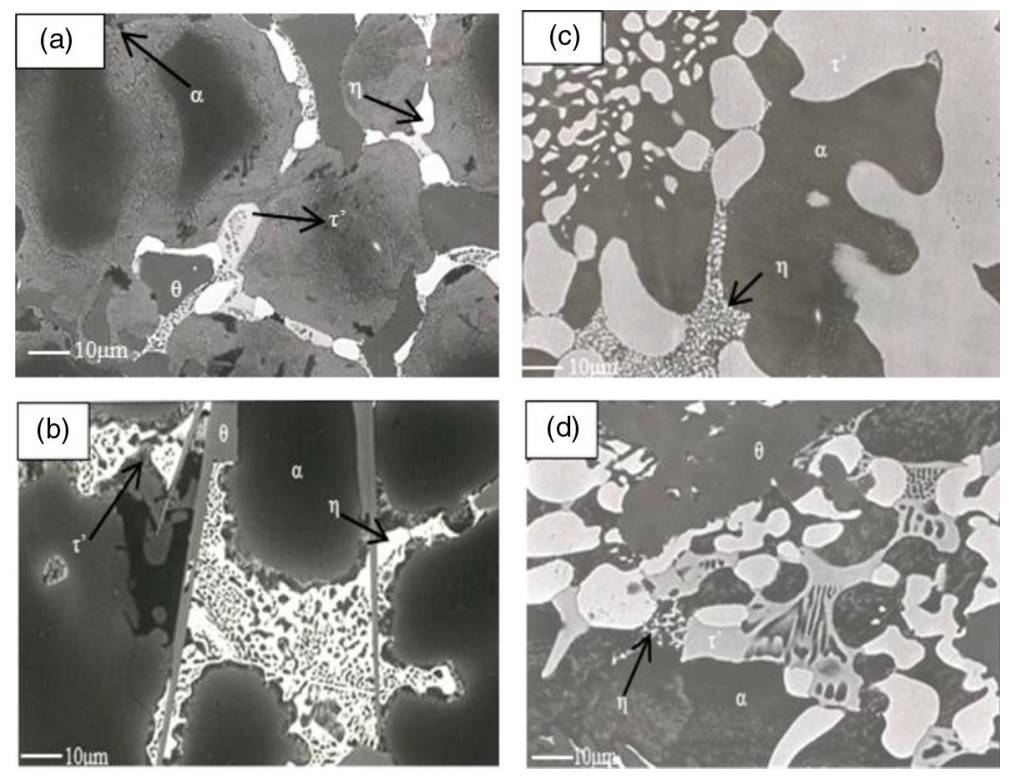

Figura 3. Microfotografías MEB de diferentes muestras en estado de colada: a) muestra M2 (Serie 1), (b) muestra M7 (Serie 1); c) muestra M10 (Serie 2) y d) muestra M15 (Serie 2).

La fase $\varepsilon$ aparece en las muestras M1 a M3, pero cabe señalar que esta fase, de acuerdo a la literatura, está involucrada en la transformación de las
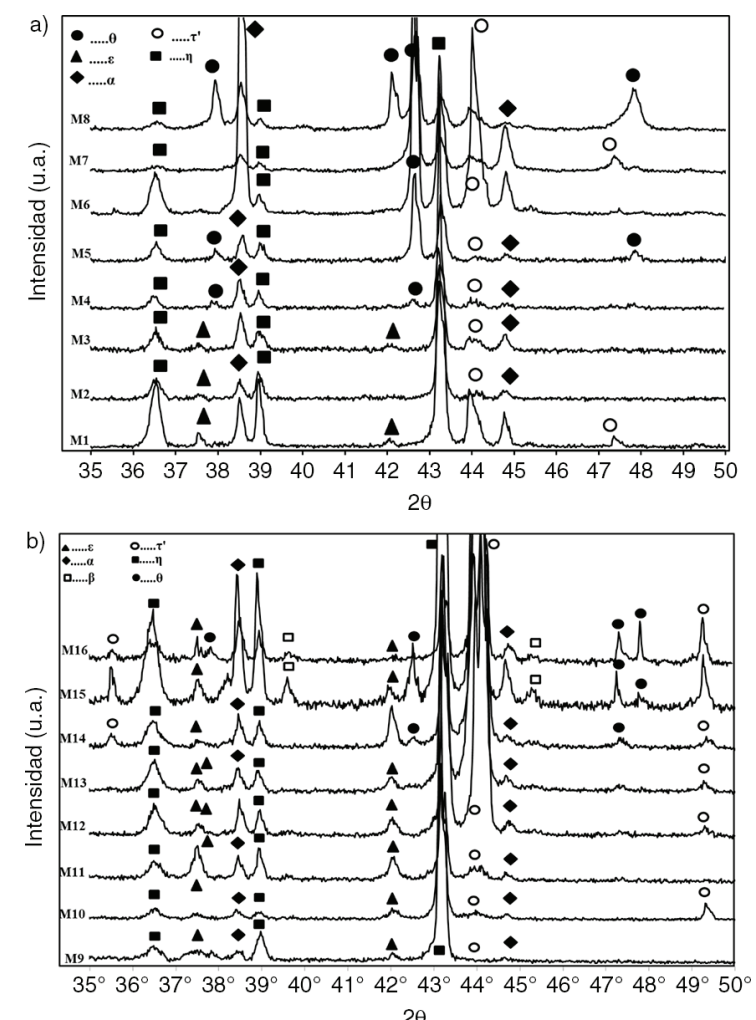

Figura 4. Difractogramas de las muestras después del proceso de homogeneización. a) Serie 1 y b) Serie 2. cuatro fases donde $\eta+\varepsilon \rightarrow \eta+\tau$ (Effenberg, 2005), por lo que tenderá a desaparecer con el tiempo. Por otra parte, la fase $\varepsilon$ desaparece en las muestras de M4 a M8 y en su lugar aparece la fase $\theta$, que tiene una mayor estabilidad dimensional (Savaskan et al., 2004).

En el caso de las muestras de la serie 2, se observa una mayor presencia de la fase $\varepsilon$, que está presente en las muestras M9 a M13. En las muestras M15 a M16 aparecen las fases $\theta$ y $\beta$. Esta última de acuerdo a Aragón et al., (2007b), se presenta después de un proceso de templado y desaparece después de unos cuantos minutos a temperatura ambiente. La fase $\beta$, se mantuvo después del proceso de homogeneizado de 180 horas, lo que indica que el alto contenido de $\mathrm{Cu}$ y Al provoca la retención de esta fase.

En la Figura 1, se puede observar que a $350{ }^{\circ} \mathrm{C}$, las muestras M2 y M15 tienen las mismas fases, como queda demostrado en los diagramas de difracción. La cantidad volumétrica de cada una de las fases es distinta. El aumento en la cantidad volumétrica de la fase $\theta$ provoca un aumento en la dureza en todas las muestras.

La Figura 5 presenta los difractogramas de las muestras después del templado. Las muestras M1 a M4 y M9 a M11 presentan exactamente las mismas fases que después del proceso de homogeneizado. Sin embargo, comparando los difractogramas después del proceso de homogeneizado y templado, se observa un incremento en la intensidad de los picos de la fase $\tau$ y $\alpha$. Debido a que las muestras se midieron en estado sólido y no en polvo, se observa la presencia de textura, pero de una forma cualitativa se puede observar que sí existe un aumento en esta 


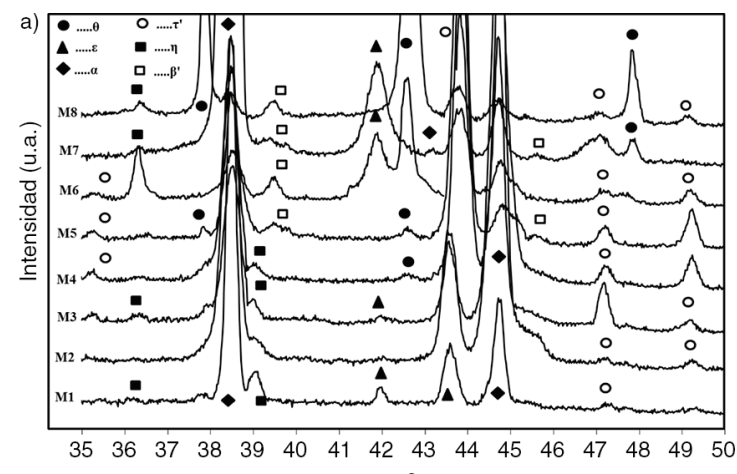

$2 \theta$

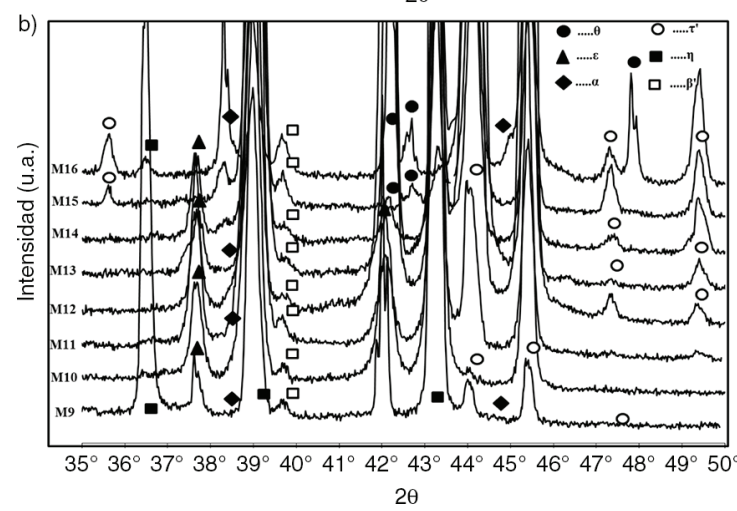

Figura 5. Difractogramas de las muestras después del proceso de templado. a) Serie 1 y b) Serie 2.

fase así como de su dureza. Cuando se aumenta la cantidad de $\mathrm{Cu}$ en más de un $15 \%$ en peso, se retiene la fase $\beta$, como se observa en las muestras de M5 a M8, mientras que en las muestras M12 y M13 se retiene la fase $\theta$. En todas estas muestras existe un incremento en el número de fases, lo que explica el aumento de la dureza. Para las muestras de la serie 2 se observa un mayor incremento en la intensidad del pico de la fase $\beta$, lo que supone una mayor cantidad de $\mathrm{Cu}$ y $\mathrm{Al}$, como se observa en las muestras M14 a M16. Desde el punto de vista de transformaciones de fases (Zhu et al., 2001), la fase $\tau^{\prime}$ se forma de acuerdo a la siguiente reacción: $\alpha+\varepsilon \rightarrow \eta+\tau^{\prime}$. Otra reacción de fases posible es la descomposición de la fase $\beta$ de acuerdo a la siguiente reacción: $\beta \rightarrow \alpha+\eta$.

La Figura 6 muestra las micrografías MEB de las muestras M2 (a-b) después del proceso de homogeneizado y M8 (c-d) después del proceso de templado, a diferentes aumentos. No se distingue restos de la estructura dendrítica y se puede observar, al igual que en el caso de las muestras de colada, que la fase $\theta$ se incrementa proporcionalmente con el aumento de $\mathrm{Cu}$ y $\mathrm{Al}$. La matriz de la muestra M2 (Fig. 6b), presenta una mezcla de las fases $\eta$ y $\alpha$, así como concentrados de la fase $\tau$. En la muestra M8, tanto en las microfotografías MEB como en los difractogramas, se observa la fase $\theta$, que se encuentra en mayor proporción. La matriz está compuesta por las fases $\eta$ y $\alpha$, teniendo esta última una mayor proporción en la muestra M8, como indica el oscurecimiento de la matriz.

\subsection{Dureza}

La dureza obtenida en cada serie, tanto en estado de colada como después del proceso de homogeneizado y templado, presenta una tendencia lineal al crecimiento en función de la fracción atómica de cobre (Fig. 7). El coeficiente de correlación $\mathrm{R}^{2}$ varía entre 0,82 y 0,97 . En la Tabla 3 se recogen las
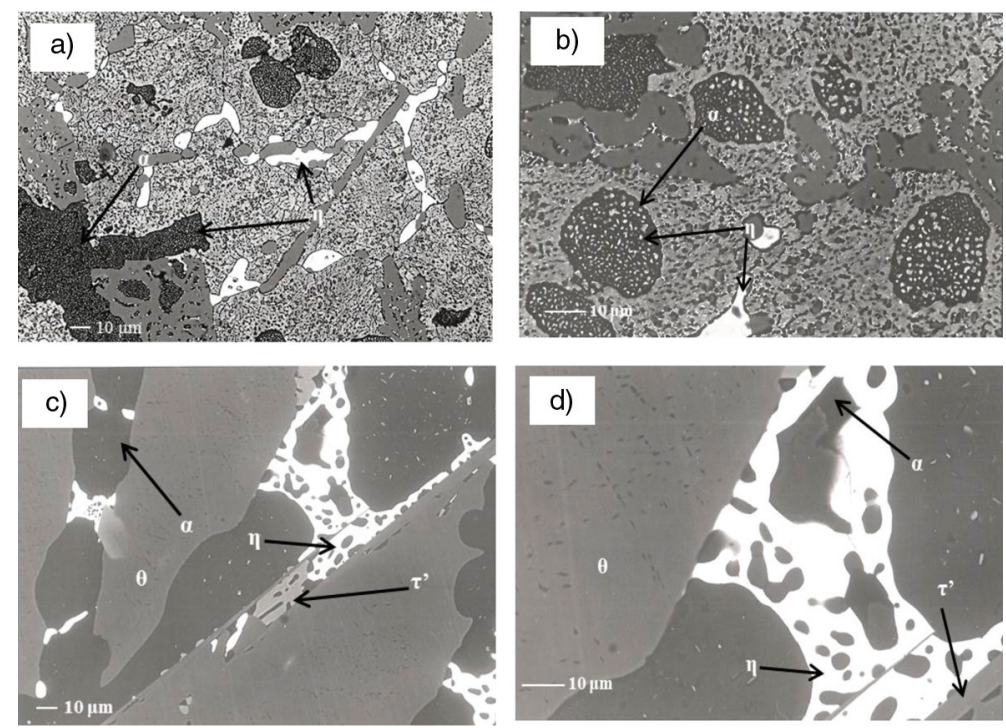

Figura 6. Microfotografías MEB después del proceso de homogeneizado y templado. a) M2 a 400X; b) M2 a 1000X; c) M8 a 400X y d) M8 a 1000X. 

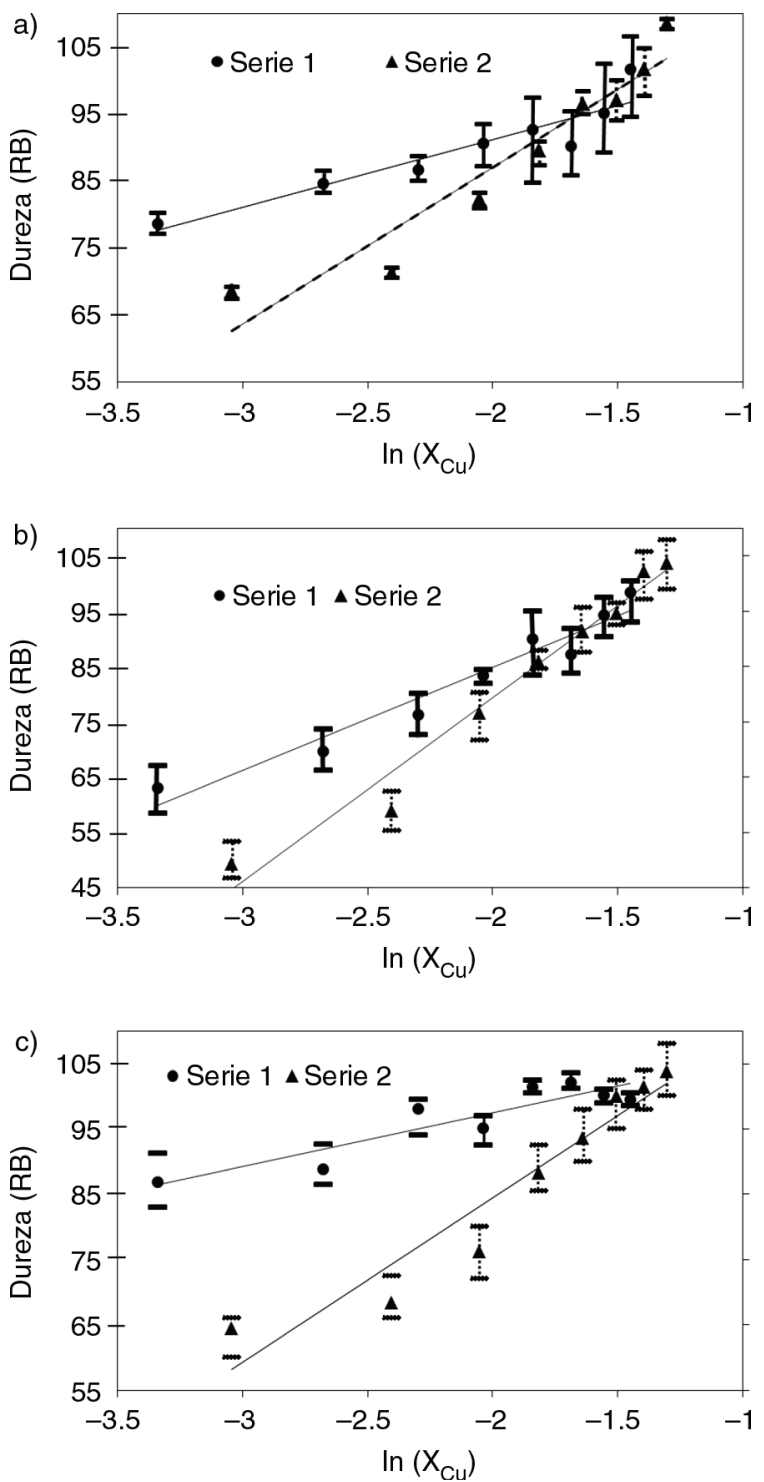

Figura 7. Gráficos de dureza de las muestras de las series 1 y 2 obtenidos con diferentes tratamientos térmicos: a) estado de colada; b) después del proceso de homogeneización y c) después del proceso de templado.

ecuaciones que relacionan la dureza y la fracción atómica de cobre para cada tratamiento y serie.

Las ecuaciones anteriores sirven para predecir la dureza de cualquier aleación cuya composición se encuentre en la línea de cada serie, como ya se había demostrado en trabajos anteriores (Villegas et al., 2011).

Se puede asumir que en general, un aumento en el porcentaje de la fase $\theta$ incrementa la dureza. Cuando la fase $\theta$ está acompañada de la fase $\beta$, como es en el caso de las muestras templadas, la dureza se incrementa aún más. Sin embargo, cuando las fases $\theta$ y $\beta$ vienen acompañas de la fase $\varepsilon$, la dureza disminuye. Es por esta razón que las muestras de la
TABLA 3. Ecuaciones representativas de la variación de la dureza en función del contenido atómico de cobre para las distintas aleaciones y tratamientos térmicos

\begin{tabular}{lcc}
\hline \multicolumn{3}{c}{ Serie 1 } \\
\hline Tratamiento térmico & Ecuación & $\mathbf{R}^{\mathbf{2}}$ \\
\hline Colada & $\mathrm{HB}=10,12 \ln \mathrm{X}_{\mathrm{Cu}}+111,38$ & 0,8673 \\
Homogeneizado & $\mathrm{HB}=18,617 \ln \mathrm{X}_{\mathrm{Cu}}+122,12$ & 0,9464 \\
Templado & $\mathrm{HB}=8,1666 \ln \mathrm{X}_{\mathrm{Cu}}+113,81$ & 0,8197 \\
\hline \multicolumn{3}{c}{ Serie $\mathbf{2}$} \\
\hline Tratamiento térmico & Ecuación & $\mathbf{R}^{\mathbf{2}}$ \\
\hline Colada & $\mathrm{HB}=23,334 \ln \mathrm{X}_{\mathrm{Cu}}+133,63$ & 0,9169 \\
Homogeneizado & $\mathrm{HB}=33,326 \ln \mathrm{X}_{\mathrm{Cu}}+146,05$ & 0,9704 \\
Templado & $\mathrm{HB}=25,211 \ln \mathrm{X}_{\mathrm{Cu}}+134,83$ & 0,9186 \\
\hline
\end{tabular}

$\mathrm{R}^{2}$ : Coeficiente de regresión lineal.

serie 1 tienen en promedio mayor dureza que las muestras de la serie 2, para un tratamiento térmico similar en ambas series.

Comparando los tratamientos térmicos entre sí (colada, homogeneizado y templado), se puede observar que para las muestras de la serie 1, en casi todos los casos, la dureza después del templado es mayor que después de colada y homogeneizado. Al realizar un análisis de los difractogramas correspondientes, se puede observar un incremento en la fracción de la fase $\alpha$. Respecto a la serie 2, se observa una disminución de la dureza en estado de templado con respecto al estado de colada y también se puede apreciar un incremento de la fracción de la fase $\alpha$ (Muestras M9 a M16 en estado de colada).

\subsection{Predicción de la dureza}

A partir de la ecuación de dureza de cada serie y para cada tratamiento (Tabla 3 ), es posible predecir la dureza de cualquier aleación cuya composición esté comprendida en el diagrama ternario entre la serie 1 y 2.

Para ello, se debe recordar que las ecuaciones (1) y (2) proporcionan cada una de las composiciones de la serie 1 y 2 . Cuando estas ecuaciones se representan en un plano cartesiano (Fig. 8), donde el eje "X" representa la fracción atómica de $\mathrm{Cu}\left(\mathrm{X}_{\mathrm{Cu}}\right)$ y el eje "Y" la fracción atómica de $\mathrm{Zn}\left(\mathrm{X}_{\mathrm{Zn}}\right)$, se pueden obtener las pendientes de cada serie. Para pronosticar la dureza de cualquier composición dentro de estas dos líneas, es necesario además, conocer el ángulo que tiene esta aleación con respecto al eje. El ángulo de cada serie está representado por $\Omega 1$ y $\Omega 2$.

Para determinar el ángulo, se representa un punto virtual, que corresponde a la intersección de las dos líneas de cada serie indicadas anteriormente. El punto de intersección obtenido es: $45,26 \%$ at. $\mathrm{Cu}$; $37,64 \%$ at. $\mathrm{Zn}$ y se le denomina virtual debido a que no puede existir físicamente. 

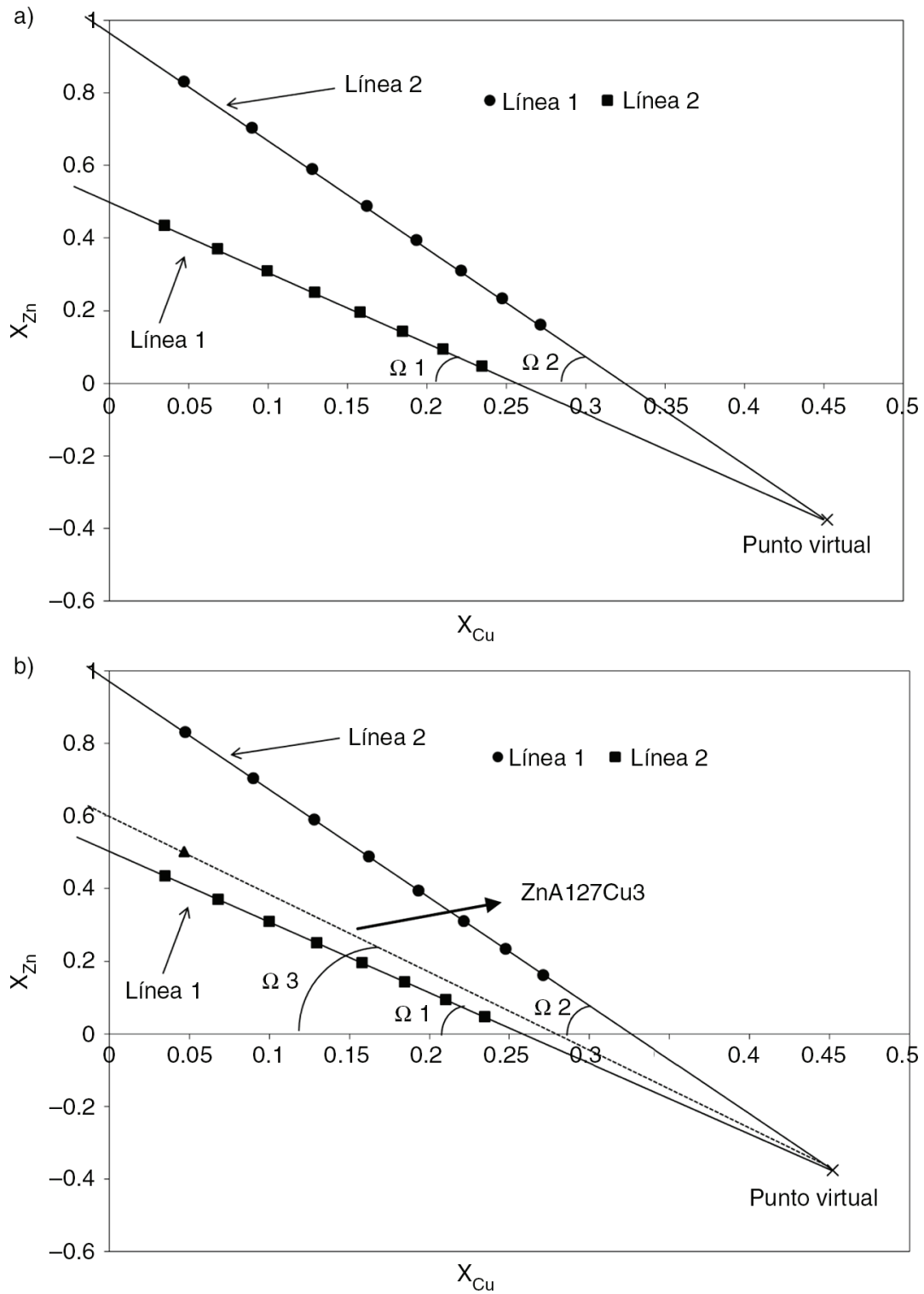

FIgURA 8. Relaciones $\mathrm{X}_{\mathrm{Zn}}$ vs. $\mathrm{X}_{\mathrm{Cu}}$ obtenidas mediante la aplicación de las ecuaciones (1) y (2). Representación en un plano cartesiano (a) y aplicación a la aleación ZnAl27Cu3 (b).

A modo de ejemplo, podemos considerar la muestra $\mathrm{ZnA} 127 \mathrm{Cu} 3$ que contiene $50,54 \%$ at. $\mathrm{Zn}$; $2,23 \%$ at. Cu y el resto $\mathrm{Al}$ (Savaskan et al., 2003). La composición de esta muestra vista en el plano cartesiano, es tan solo un punto, cuando se une al punto virtual se forma una línea recta, con la cual se puede medir la pendiente y determinar el ángulo (Fig. 8b). El resultado obtenido para el ángulo es $63,99^{\circ}$.

Tanto la pendiente de la línea 1 como de la línea 2 tiene relación directa con las pendientes obtenidas en las ecuaciones de dureza presentadas en la Tabla 3. Por lo tanto, es posible obtener por interpolación la pendiente de dureza que le corresponde a la muestra $\mathrm{ZnAl} 27 \mathrm{Cu} 3$ en estado de colada. Los resultados se muestran en la Tabla 4.
Para predecir la dureza de la muestra $\mathrm{ZnA1} 27 \mathrm{Cu} 3$ hay que conocer también su ordenada al origen. Para ello, es necesario tener un punto en el cual la dureza de todas las composiciones, comprendidas

TABLA 4. Valores de los ángulos de cada serie y de la muestra a pronosticar $(\mathrm{ZnAl} 27 \mathrm{Cu} 3)$

\begin{tabular}{lcc}
\hline Muestra & $\begin{array}{c}\text { Angulo del porcentaje } \\
\text { de cada elemento }\end{array}$ & $\begin{array}{c}\text { Angulo en el gráfico de } \\
\text { dureza en estado de colada }\end{array}$ \\
\hline Línea 1 & $62,78^{\circ}$ & $84,74^{\circ}$ \\
ZnA127Cu3 & $63,99^{\circ}$ & $\begin{array}{l}84,80^{\circ} \text { valor } \\
\text { por interpolación } \\
\end{array}$ \\
Línea 2 & $71,47^{\circ}$ & $87,55^{\circ}$
\end{tabular}


TABLA 5. Resultados comparativos de la dureza determinada por Savaskan et al., (2003) y los valores experimentales obtenidos en este trabajo, para diversas aleaciones

\begin{tabular}{lccc}
\hline Muestra & $\begin{array}{c}\text { Savaskan } \\
\text { et al., (2003) }\end{array}$ & $\begin{array}{c}\text { Presente } \\
\text { trabajo }\end{array}$ & Error (\%) \\
\hline ZnA127Cu1 & 64,74 & 59,94 & 7,41 \\
ZnA127Cu2 & 66,21 & 66,98 & 1,15 \\
ZnA127Cu3 & 67,15 & 71,04 & 5,48 \\
ZnA127Cu4 & 68,50 & 73,91 & 7,32 \\
ZnA127Cu5 & 70,75 & 76,12 & 7,05 \\
& \multicolumn{3}{c}{ Error promedio $=5,68$} \\
\hline
\end{tabular}

TABLA 6. Resultados comparativos de la dureza obtenidos por Ciach et al., (1969) y los valores experimentales obtenidos en este trabajo, para diversas aleaciones

\begin{tabular}{lccc}
\hline Muestra & $\begin{array}{c}\text { Ciach } \text { et al., } \\
\text { (1969) }\end{array}$ & $\begin{array}{c}\text { Presente } \\
\text { trabajo }\end{array}$ & Error (\%) \\
\hline $\mathrm{AlZn} 78 \mathrm{Cu} 1$ & 73 & 66,99 & 8,24 \\
$\mathrm{AlZn} 78 \mathrm{Cu} 2$ & 78 & 73,12 & 6,25 \\
$\mathrm{AlZn} 78 \mathrm{Cu} 3$ & 81 & 76,53 & 5,52 \\
& \multicolumn{3}{c}{ Error promedio $=6,67$} \\
\hline
\end{tabular}

entre la serie 1 y 2 , se interceptan. La Figura $7 \mathrm{a}$ muestra el gráfico de dureza, cuando las muestras están en estado de colada. Éstas se interceptan en $18,57 \%$ at. $\mathrm{Cu}$, cuando la dureza es de $94,34 \mathrm{RB}$, lo que da como resultado una ordenada en el origen de 114,11 RB. Por lo tanto, la ecuación representativa de variación de la dureza para la muestra ZnAl27Cu3 se expresa en la ecuación (3), que permite obtener un valor de dureza de 69,45 RB.

$$
\mathrm{HB}=\tan (85,13) \ln \mathrm{X}_{\mathrm{Cu}}+114,11
$$

Para esta aleación, el valor de la dureza obtenido por Savaskan et al., (2003) es de 67,15 RB lo que da un error de $5,48 \%$.

La Tabla 5 presenta el análisis entre los valores obtenidos por Savaskan et al., (2003) y los obtenidos con el método propuesto en este trabajo. Se observa que el error máximo es de 7,41\% y el mínimo de $1,15 \%$.

Aplicando el método propuesto en este trabajo a las aleaciones estudiadas por Ciach et al., (1969), se obtienen los resultados que aparecen en la Tabla 6. El error promedio es de un 6,67\% debido en parte, a la preparación de las muestras, que es un tanto diferente en ambas metodologías.

\section{CONCLUSIONES}

El aumento de los contenidos de $\mathrm{Cu}$ y $\mathrm{Al}$ produce la retención de fases $\beta$ y $\theta$, lo cual origina un aumento en la dureza. En cambio, cuando aparecen las fases $\beta$ y $\varepsilon$ la dureza disminuye.
El programa Thermo-Calc permite conocer las fases existentes en cada una de las muestras estudiadas. Los resultados obtenidos son coherentes con las fases presentes en el diagrama de Börnstein, lo que despeja la discrepancia entre el diagrama de Börnstein y Jan.

Las muestras estudiadas presentan las mismas fases que las obtenidas mediante Thermo-Calc más las fases $\varepsilon$, y $\theta$, que tienden a desaparecer conforme se acercan a un estado de equilibrio. Estos resultados, fueron coherentes con los obtenidos en el estudio mediante Difracción de RX que mostraron que en estado de colada y homogeneizado se observó una disminución de la intensidad de los picos de las fases $\varepsilon$, y $\theta$.

La metodología presentada en este trabajo, permite obtener una buena aproximación de la dureza que tendrá cualquier aleación cuya composición química esté comprendida entre las dos series estudiadas en este trabajo, con un error constante, cuyo valor depende de las condiciones en las que se realice el pronóstico.

La metodología expuesta en este trabajo, puede extrapolarse a otros sistemas ternarios para predecir sus propiedades mecánicas.

\section{REFERENCIAS}

Aragón, J.A., Miranda, J.R., Hilerio, I., Muñoz, D., Hernández, R., Cortés, V., Altamirano, A. (2007a). Compuestos de matriz metálica rica en $\mathrm{Zn}$, con alto contenido de Al y componentes estructurales de compuestos intermetálicos de $\mathrm{Cu}-\mathrm{Zn}$ y $\mathrm{Cu}-\mathrm{Al}$ particulados. Rev. Mex. Fís. 53 (2), 105-113. http://rmf.smf.mx/pdf/rmf/53/2/ 53_2_105.pdf.

Aragón, J.A., Miranda, J.R., García, A. (2007b). Obtención de una microestructura nueva en la aleación $\mathrm{Zn}-40 \%$ at. Al-1,5\% at. Cu. Rev. Mex. Fís. 53 (3), 149-158. http://rmf. smf.mx/pdf/rmf/53/3/53_3_149.pdf.

Bai-quing, X. (1999). Present and development of spray forming technology. Chi. J. Ra. Met. 11 (6), 26-37.

Börnstein, L. (2005). Ternary Alloy System Phase Diagrams, Crystallographic and Thermodynamic Data, Ed. Springer, Vol. 1, 11, New York, USA, pp. 183-205.

Cáceres, C.H., Lescano, E.E. (1990). The growth of artificial voids during superplastic deformation of a $\mathrm{Zn}-\mathrm{Al}-\mathrm{Cu}$ alloy. Mater. Sci. Eng. A, 128 (2), 147-154. http://dx.doi.org/ 10.1016/0921-5093(90)90223-P.

Cáceres, C.H., Silvetti, S.P. (1987). Cavitation damage in the superplastic $\mathrm{Zn}-22 \% \mathrm{Al}-0.5 \% \mathrm{Cu}$ alloy. Acta Metall. 35 (4), 897-906. http://dx.doi.org/10.1016/0001-6160(87)90168-4.

Cenoz, E., Fernández, C. (2007). Influencia de la composición y el tratamiento térmico en las propiedades mecánicas de aleaciones de bronce al aluminio. Rev. Metal. 43 (4), 272-283. http://dx.doi.org/10.3989/revmetalm.2007.v43.i4.73.

Chang, S.C., Jahn, M.T., Wan, C.M., Lee, J.Y., Hsu, Y.T.K. (1976). The determination of tensile properties from hardness measurements for AI - Zn-Mg alloys. J. Mater. Sci. 11 (4), 623-630. http://dx.doi.org/10.1007/BF01209447.

Ciach, R., Krol, J., Wegryn, K. (1969). Studies on four phases transformation in AlZn78 alloy containing 1-3 per cent of copper. Bulletin de L'Academie Polonaise des Sciences 17 (4), 371-378.

Effenberg, G. (2005). Ternary Alloys Systems, Part 2. Selected Systems form Al-Cu-Fe to Al-Fe-Ti, Ed. Springer, Vol. 1, New York, USA, pp. 329-358

Gelfi, M., Bontempi, E., Pola, A., Roberti, R., Rollez, D., Depero, L.E. (2004). Microstructural and Mechanical 
Properties of Zinc Die Casting Alloys. Adv. Eng. Mater. 6 (10), 818-822. http://dx.doi.org/10.1002/adem.200400087.

Ghosh, G., Humbeek, J.V. (1992). Ternary Alloys, Ed. VCH, 1, 5, New York, USA, pp. 92-112.

Malvano, M.L., Marantonio, M. (1911). Researches on the Constitution of Al. Gazz Chim. Ital. 41, 282-297.

Mondolfo, L.F. (1976). Metallography of aluminium Alloys. Ed. John Wiley \& Son, Vol. 1, New York, USA, pp. 518-520.

Ramos, M., Martínez, E. Torres, G. (2012). Superplastic behavior of $\mathrm{Zn}-\mathrm{Al}$ eutectoid alloy with $2 \%$ Cu. J. Mater. Sci. 47 (17), 6206-6212. http://dx.doi.org/10.1007/s10853-012-6494-z.

Sandoval A., Negrete J., Torres G. (2000). Influencia de las fases $€$ y $\tau^{\prime}$ en las propiedades mecánicas del zinalco V. Rev. Mex. Fis. 46, 361-372. http://rmf.smf.mx/pdf/rmf/46/4/46_4_361.pdf.

Savaskan, T., Murphy, S. (1983). Creep behaviour of $\bar{Z} n-A l-C u$ alloys. Metallkd 74 (2), 76-82.

Savașkan, T., Pürçek, G., Heikimoğlu, P. (2003). Effect of copper on the mechanical and tribological properties of ZnAl27 based alloys. Tribol. Lett. 15 (3), 257-263.http://dx.doi. org/10.1023/A:1024817304351.
Savașkan, T., Heikimoğlu, P., PÜRçEK, G. (2004). Effect of copper content on the mechanical and sliding wear properties of monotectoid-based zinc-aluminium-copper alloys. Tribol. Int. 37 (1), 45-50. http://dx.doi.org/10.1016/S0301-679X(03) 00113-0.

Villars, P., Prince, A., Okamoto, H. (1997). Handbook of Ternary Alloy Phase Diagrams, Ed. ASM International, the Materials Society, New York, USA, 3416.

Villegas, J.D., López, V.M., Ita, A., Saucedo, M.L. (2011). Assessment of hardness in As Cast and homogenized $\mathrm{Zn}$ Al-Cu Alloys. Mater. Trans. 52 (8), 1581-1584.

Wu, M.W., Hwang, K.S., Huang, H.S. (2007). In-Situ Observations on the Fracture Mechanism of Diffusion-Alloyed NiContaining Powder Metal Steels and a Proposed Method for Tensile Strength Improvement. Metall. Mater. Trans. A 38 (7), 1598-1607. http://dx.doi.org/10.1007/s11661007-9201-y.

Zhu, Y.H. (2001). Phase transformations of eutectoid $\mathrm{Zn}-\mathrm{Al}$ alloys. J. Mat. Sci. 36 (16), 3973-3980. http://dx.doi.org/ 10.1023/A:1017978407093. 